\title{
Size-dependent magnetic properties of Iron Carbide nanoparticles embedded in a carbon matrix
}

\author{
E.P.Sajitha ${ }^{1}$, V. Prasad ${ }^{1}$, S.V.Subramanyam ${ }^{1}$, Ajay Kumar \\ Mishra $^{2}$, Subhajit Sarkar ${ }^{2}$ and Chandrahaas Bansal ${ }^{2}$ \\ ${ }^{1}$ Department of Physics, Indian Institute of Science, Bangalore, India, 560012 \\ 2 School of Physics, University of Hyderabad, Hyderabad, 500 046, India \\ E-mail: sajitha@physics.iisc.ernet.in
}

\begin{abstract}
Here we report on the magnetic properties of iron carbide nanoparticles embedded in a carbon matrix. The granular distribution of nanoparticles in an inert matrix, of potential use in various application, were prepared by pyrolysis of organic precursors using thermal assisted chemical vapour deposition method. By varying the precursor concentration and preparation temperature, compositions with varying iron concentration and nanoparticle sizes were made. Powder X-ray diffraction, Transmission Electron Microscopy and Mössbauer spectroscopy studies revealed, the nanocrystalline iron carbide $\left(\mathrm{Fe}_{3} \mathrm{C}\right)$ presence in the partially-graphitized matrix. The dependence of magnetic properties on the particle size and temperature $(10 \mathrm{~K}<\mathrm{T}<300$ K) were studied using Superconducting Quantum Interference Device Magnetometery. Based on the effect of surrounding carbon spins, the observed magnetic behaviour of the nanoparticle compositions, such as temperature dependence of magnetization and coercivity, can be explained.
\end{abstract}

PACS numbers: $75.50 . \mathrm{Tt}, 76.80 .+\mathrm{y}, 75.60 . \mathrm{Ej}$

Keywords: Nanocrystalline Magnetic materials, Mössbauer Spectroscopy, Magnetization

\section{Introduction}

The recent interest in magnetic nanostructures lies in the emergence of novel magnetic and transport properties with the reduction of size. As the dimension approaches the nanometer length scale, interesting size-dependence properties like enhanced coercivity $[1,2,3]$, enhanced magnetization $[4,5,6]$, superparamagnetism [7] etc. are seen. The interest in nano-sized materials have spread to other disciplines of physics, chemistry and medicine due to the possible technological application associated with them apart from the fundamental aspects $[8,9]$.

The macroscopic physical properties of the magnetic nanoparticle species depends on the size, shape and morphology of the constituents dispersed in a non-magnetic metallic or insulating medium and on the strength of exchange coupling between the particles $[1,2,3,10,11,12]$. Among the various materials, carbon is an attractive 
candidate to wrap the ferromagnetic transition metal and their carbides, providing thermal stability and control over the particle size distribution [10].

Carbon-iron ( $\mathrm{C}: \mathrm{Fe}$ ) based systems are of growing interest due to their improved magnetic properties as well as in their potential application as sensors, catalyst, and in the potential reduction of the cost required to produce bulk quantities $[13,14,15,16]$. In particular, nanocomposites of iron carbides, such as the cementite phase $\mathrm{Fe}_{3} \mathrm{C}$, are further suited to diverse technological exploitations [17] due to their enhanced mechanical properties [18] and importance in ferrous metallurgy [19]. Nanocomposites of carbon containing iron nanoparticles exhibit the properties of both constituents, i.e., magnetic as well as conducting, have proved to be a useful filler material for electromagnetic shielding applications in the form of coating or sheaths [20, 21].

The focus of this paper is on the magnetic properties of $\mathrm{Fe}_{3} \mathrm{C}$ nanoparticles effected by the particle size and matrix (carbon) morphology. Nanoparticle composites with varying particle size and concentration were prepared by the pyrolysis of organic precursors in known ratios using thermal assisted chemical vapour deposition (CVD) method. The results show enhanced coercivity in all the prepared compositions along with enhanced magnetic moment in the samples containing ultrafine nanoparticles.

\section{Experiment}

Synthesis of iron carbide nanoparticles in the carbon matrix was accomplished by thermal assisted CVD of organic precursors, maleic anhydride and ferrocene, in known ratio at elevated temperature of $900^{\circ} \mathrm{C}$ and $980^{\circ} \mathrm{C}$. The details of the experimental procedures have been described elsewhere [22]. Here it is sufficient to say that pyrolysis of the organic mixture containing maleic anhydride and ferrocene in different weight percentages was carried out in a quartz tube (10 $\mathrm{mm}$ diameter and $500 \mathrm{~mm}$ length), one end closed and other end attached to an external bladder. In the text, designations followed for the C:Fe compositions are CFe05900, CFe10900, CFe05980 and CFe10980 where the first two numbers indicate the ferrocene molecular weight percentage in the precursor and the last three numbers indicate the preparation temperature.

The crystallographical phases were determined using X-ray diffraction (XRD) patterns taken with a Philips X'pert diffractometer, using $\mathrm{CuK} \alpha$ radiation. The morphology of all the compositions has been studied by high resolution transmission electron microscopy (HRTEM) in a FEI Technai F30 electron microscope. Mössbauer spectra [23] were obtained using a microprocessor controlled conventional constant acceleration Mössbauer spectrometer and a $15 \mathrm{mCi}{ }^{57} \mathrm{Co}$ source in Rhodium matrix. The spectrometer was calibrated by collecting spectrum of a standard $\alpha$-Fe foil at room temperature. Magnetization measurements $\mathrm{M}(\mathrm{T}, \mathrm{H})$ in applied magnetic fields between $\pm 5 \mathrm{~T}$ and for temperatures ranging from 10 to $300 \mathrm{~K}$, were performed in a Quantum Design (superconducting quantum interference device) SQUID magnetometer. To determine the coercivity, samples were first cooled in zero applied magnetic field from room temperature down to the measuring temperature and then the hysteresis loops 
were measured in the presence of magnetic field. The microanalyses of the compositions were carried out using ThermoFinnigan FLASH EA 1112 CHNS analyser.

\section{Results and discussions}

\subsection{Structural measurement}

Table 1 displays the initial molecular weight percentage of ferrocene taken with maleic anhydride and the final iron and hydrogen percentage in the prepared C:Fe compositions. The iron content in the compositions are estimated from open air oxidization of the powders. The percentage of hydrogen in the compositions are estimated from the microanalyses experiment. For a given percentage of ferrocene in the precursor, the iron content decreases with increase in preparation temperature. It is reported [24] that the growth modes of Fe clusters depends on the initial supersaturation and the density of iron vapor. At low temperature, the nucleation rate is higher and more clusters are formed which eventually collides and forms larger ones. With increasing temperature the nucleation rate decreases and the growth of clusters are mainly dominated by surface growth. Another cause for the decrease in iron content would be the evaporation of small iron clusters at higher temperature. This phenomenon is explained with regard to the factors effecting the cessation of nanotube growth as a function of temperature and precursor concentration [25].

The XRD pattern (Fig.1) clearly shows the orthorhombic crystal structure of iron carbide $\left(\mathrm{Fe}_{3} \mathrm{C}\right)$ in as-prepared C:Fe compositions [22]. The diffraction peak at $26.4^{\circ}$, assigned to graphite (002) plane is also seen. As the preparation temperature increases, the intensity and the width of graphite peak (002) concurrently decreases, indicating the decrease in graphitization. For graphite-like carbon the degree of graphitization is estimated from the interlayer spacing $d$, determined from the position of the $G(002)$ diffraction line, using the Maire and Mering formula [26],

$$
d=3.354+0.086(1-g)
$$

where $g$ is the graphitization percentage. The interlayer spacing $d$, degree of graphitization $g$ and calculated graphite crystallite $L_{C}$ (using Scherrer formula) are also tabulated in Table 1 . In the prepared compositions the yield of carbon and the degree of graphitization has a direct dependence on the iron activity in the given temperature range. There has been earlier reports on maximal iron activity in certain temperature range and extremely low activity as the temperature increases [27]. The decomposition temperature, together with the ferrocene percentage has a marked effect on the morphology and final product of the compositions.

The microstructures of the compositions with lower Fe content were very similar as shown in the TEM micrographs, Fig. 2. The dark oval shaped core corresponds to metallic iron carbide whereas the surrounding light grey layer is the carbon matrix. The histograms of the particle size distribution, obtained from the TEM images, were fitted using log-normal distribution. The fitting of CFe05980 resulted in a maxima at 11.3 
$\mathrm{nm}$ and a standard deviation, $\sigma$ of 0.37 whereas for CFe10980, the maxima appeared at $11.7 \mathrm{~nm}$ with $\sigma=0.27$. The distribution gave an average particle diameter $11.2 \mathrm{~nm}$ for $\mathrm{CFe} 05980$ and $12 \mathrm{~nm}$ for CFe10980. The result obtained here indicates that increase in iron percentage in the precursor did not change the average size of the particles. However, an increase in the volume density of the CFe10980 samples cannot be excluded. The TEM analysis also reveals the coalescence of primary particles, resulting in larger sizes whose symmetry departs from the singular entities [Fig. 2(b) and (f)]. As the iron content in the compositions increases, agglomerated iron carbide particles where seen in the TEM micrographs of CFe05900 and CFe10900 [22]. Even though the set time of preparation, five hours, is enough to curtail the growth of nanotubes, finite amount of hollow carbon filaments with no metal inclusions, where seen in the micrographs with lower Fe content. The electron diffraction pattern of the compositions Fig 2 (d) and (h) displays the diffused rings (002), (100) and (110) originating from the electron scattered from the carbon matrix. The reflexes from the orthorhombic $\mathrm{Fe}_{3} \mathrm{C}$ were also seen. No reflections could be assigned to $\alpha$ - $\mathrm{Fe}$ or $\mathrm{Fe}_{3} \mathrm{O}_{4}$ phases.

\subsection{Hyperfine Field}

To investigate the $\mathrm{Fe}$ atoms occupation in the crystal lattice of iron carbide, the Mössbauer measurement was carried out. The collected room-temperature Mössbauer spectra [Fig.3 and Fig.4] have been least square fitted [28] with the parameters listed in Table 2. The component with the hyperfine field around $20 \mathrm{~T}$ confirmed the presence of crystalline iron carbide in all the compositions. It is pointed out that Fe atoms in $\mathrm{Fe}_{3} \mathrm{C}$ occupy two different lattice sites, which theoretically should be fitted with two sextets with different hyperfine fields $(20.5 \mathrm{~T}$ and $20.7 \mathrm{~T})$ and same isomer shift of $0.17 \pm 0.02 \mathrm{~mm} / \mathrm{s}$ [29]. Considering that the hyperfine field and the chemical isomer shift for the two sites are close, it would be acceptable to use one sextet to identify the presence of $\mathrm{Fe}_{3} \mathrm{C}$. The nonmagnetic doublet would be attributed to superparamagnetic/ paramagnetic iron carbides and/or iron oxides.

The high noise level in the CFe05980 and CFe10980 spectra, containing lowest percentage of $\mathrm{Fe}$, is mainly due to the low recoil-free factor, $f$. The recoil-free factor $\left[f=\exp \left(-\frac{E_{\gamma}^{2}\left\langle x^{2}\right\rangle}{\hbar c^{2}}\right)\right]$ depends on the mean square vibrational amplitude $\left\langle x^{2}\right\rangle$ of the emitting or absorbing nucleus, for a given gamma energy source. In the case of small iron particles [30], it is reported that the recoil-free fraction decreases with increasing temperature for loosely packed samples. The decrease was even more prominent in the case of specimens with lower densities of Fe atoms. In order to obtain a reasonably good fit for the Mössbauer spectra, where the probe nuclei is in several different local environments, continuous distribution of static hyperfine parameters is used [Fig.4].

Effect of carbon atoms on the structure and magnetic properties of the nanoparticle species are very obvious. The reported isomer shift values are important for the interpretation of the hyperfine field values. Owing to the changes in the electron density at the nucleus accompanied by the change in spin density, which arises partly from core 
(localized) electrons i.e. the 1s, 2s and 3s electrons and partly from conduction electrons, the center of the spectra varies. This variation in the isomer shift is due to the coulomb interaction with the nuclear distribution.

\subsection{Magnetic Properties}

An extensive study is carried out to understand the qualitative effect of particle size and matrix morphology on the magnetic properties of $\mathrm{C}: \mathrm{Fe}$ compositions. The as-prepared C:Fe compositions has saturation magnetization values between 8 and $15 \mathrm{emu} / \mathrm{g}$ depending on the preparation temperature and iron concentration. The iron weight percentage in each of the prepared compositions is used to calculate its contribution to the magnetization $\left(M_{S-\text { cal }}\right)$, assuming $M_{S}$ of $\sim 140 \mathrm{emu} / \mathrm{g}$ for $\mathrm{Fe}_{3} \mathrm{C}$ at room temperature (the value increases to $\sim 169 \mathrm{emu} / \mathrm{g}$ at $0^{\circ} \mathrm{K}$ ) [31]. The experimental magnetization values, $M_{S}$, at $300 \mathrm{~K}$ and $10 \mathrm{~K}$, together with the calculated values, $M_{S-\text { cal }}$ are given in Table 3 . Comparing the tabulated values of $M_{S}$ and $M_{S-\text { cal }}$ (Table 3 ), we see that the samples prepared at $900^{\circ} \mathrm{C}$ show lower $M_{S}$ values than the calculated $M_{S-c a l}$ values. The reduction of the magnetization is a specific phenomenon in the nanosized materials due to the formation of a surface shell with spin disorder. However, for the samples prepared at higher temperature, the $M_{S}$ values are higher than the $M_{S-\text { cal }}$ values. The difference $\left(M_{S}-M_{S-c a l}\right)$ may be attributed to the contribution from magnetic carbon species present in the samples, but a deeper understancing of the underlying mechanism is necessary.

The C:Fe compositions shows a finite coercivity at room temperature for all the prepared concentrations. Fig.5 displays the hysteresis loops for the compositions at 300 and $10 \mathrm{~K}$. At $300 \mathrm{~K}$, the magnetization shows a tendency to saturate at $15 \mathrm{kOe}$ and the coercivities are in the range of 240 Oe to 360 Oe. This suggests that the effect of superparamagnetic particles on the hysteresis loop is very negligible. As the measurement temperature is lowered, the magnetization curve attains saturation at a higher field and the coercivity value increases to around $2 \mathrm{kOe}$. The slower approach to saturation at low temperature indicate a Curie-like paramagnetic contribution from the partially-graphitized carbon matrix [32]. The influences of carbon matrix on the magnetic properties of the C:Fe compositions are further emphasized later in this section. The temperature dependence of coercivity and remanence to saturation ratio $\left(M_{R} / M_{S}\right)$ for the $\mathrm{CFe} 05980$ composition are shown in Fig. 6. In magnetic granular system, consisting of a random distribution of fine magnetic particles embedded in a nonmagnetic metallic matrix, strong magnetic interactions (exchange interaction as well as dipolar interactions between particles) are expected. The exchange coupling developed through the conducting matrix and the direct exchange of close enough grains, and the dipolar interactions acts to locally align the dispersed particles parallel or antiparallel, depending on the interparticle separation and topological distribution. The complex state of interactions, which can create strong pinning centers for the core moments during the demagnetization, explains the high coercivity observed in these 
samples, higher than the value obtained from the Stoner-Wohlfarth model for spherical single domain Fe grains ( $\sim 180$ Oe at room temperature) [33]. The effect of thermal relaxation on the surface clusters is to decrease the coercivity and remanence as the temperature increases through weakening of the pinning forces at the interfaces.

Fig. 7 shows the temperature dependence of magnetization measured at $5 \mathrm{~T}$ for the different compositions. The solid line in the Fig. 7 shows a fit to the combination of Bloch's $\mathrm{T}^{3 / 2}$ law and Curie-Weiss behavior [34]. As the measuring temperature is less than $27^{\circ} \mathrm{C}\left(\mathrm{T}_{C} \sim 220^{\circ} \mathrm{C}\right.$ ), the spin-wave theory should hold quiet well for the iron carbide inclusions. Accordingly,

$$
M(T)=M_{S}\left(1-B T^{3 / 2}\right)+\frac{C}{T-\theta}
$$

where $\mathrm{B}$ is related to the spin-wave-stiffness parameter $\mathrm{D}$ by the relation $B=$ $2.62 \frac{g \mu_{B}}{M_{S}}\left[\frac{k_{B}}{4 \mu D}\right]^{3 / 2}$. The temperature dependence of magnetization shows a combination of ferromagnetic behavior and Curie-Weiss behavior. The Curie-Weiss behavior is consistent with the picture of localized carbon spins. Curie-Weiss behavior has been observed in interacting ferrofluids [35, 36, 37], different carbon forms [38], etc. where $\theta$ is a measure of the interaction strength. A negative value of $\theta$ is attributed to the presence of stronger interactions that cause aggregation and subsequent antiferromagnetic-like ordering of the spins. The fitted parameters for the C:Fe compositions are given in Table 4. For the C:Fe compositions, the Bloch's constant, $B$ is found to be larger by an order of magnitude compared to the bulk value, implying stronger dependence of magnetization with temperature. Deviation from the spin-wave theory, was reported earlier for ribbon-shaped Fe nanoparticles [39], Fe nanoparticles embedded in $\mathrm{Mg}$ and $\mathrm{MgF}_{2}$ [40], Fe-Si samples [41], Ni nanowires [42] etc.

Deviation from the spin-wave theory is seen, in the low temperature regime, with a distinctive upturn from the $\mathrm{T}^{3 / 2}$ law. In nanostructured ferromagnetic materials the high probability disorder in the spins leads to finite entropy (considered very small in bulk ferromagnetic materials), and thus the chemical potential term in BoseEinstein distribution function cannot be neglected [43], leading to subtle upturn in the magnetization curves. Including the chemical potential, term in the traditional derivation by Kittel, a more rigorous form is obtained.

$$
M(T)=M_{S}\left[1+\frac{1}{8 \pi^{3 / 2} S_{0}}\left(\frac{k T}{2 S_{0} J}\right)^{3 / 2} \sum_{n=1}^{\infty} \frac{e^{n S\left[T_{B E}-T\right] / k T}}{n^{3 / 2}}\right]
$$

Here $\mathrm{S}_{0}$ is the spin, $\mathrm{J}$ is the coupling constant, $\mathrm{T}_{B E}$ the Bose-Einstein condensation temperature and $\zeta=S\left(T_{B E}-T\right)$ the chemical potential. For $T \leq T_{B E}, \zeta$ is zero (as in bulk system) and the summation reduces to 2.612 and for $T \gg T_{B E}$, the summation becomes a constant and the temperature dependence of magnetization follows $T^{3 / 2}$ law. In the intermediate temperature range the value of the summation decreases abruptly with the increase in temperature, signaturing a sharp drop in magnetization value. In our compositions it is difficult to ascertain any deviation from the spin wave theory because of the Curie-Weiss interaction presence. 
From the fitted values of $C$, the localized carbon spin concentration $N_{l o c}$ (Table 4), is estimated using the relation,

$$
C=\frac{N_{l o c} S(S+1) g^{2} \mu_{B}}{3 k_{B}}
$$

Here we have taken the spin quantum number $\mathrm{S}=1 / 2$ and $g=2$. Interaction between the localized spins which are not in direct contact, is mediated by the ferromagnetic clusters $\left(\mathrm{Fe}_{3} \mathrm{C}\right)$ via a Ruderman-Kittel-Kasuya-Yosida (RKKY) type interaction [37, 44].

Fig. 8 shows the zero-field cooled $\left(M_{Z F C}\right)$ and field-cooled $\left(M_{F C}\right)$ magnetization as a function of temperature in an applied field of 200 Oe for the prepared C:Fe compositions. For the ZFC measurement, the sample is cooled in the absence of an external magnetic field and then the magnetization data taken in the presence of external field with increasing temperature. For the FC measurement the sample was cooled in the presence of external magnetic field and then the magnetization data taken with increasing temperature. Here the $M_{F C}$ value, increases almost linearly with decreasing temperature, demonstrating significant irreversibility in the magnetic system. The $M_{Z F C}$ value increases with increasing temperature, with a very broad maximum around $300 \mathrm{~K}$. If the temperature of the nanoparticle composition is decreased in the zero external magnetic field state, zero magnetization is observed. As soon as an external magnetic field is applied the energy per unit volume of the system becomes

$$
E=K \sin ^{2} \theta-\mu H^{\prime} \cos \phi
$$

where $\theta$ is the angle between the magnetic moment of the particle and the easy axis for magnetization, $\mu$ is the magnetic moment of the particle per unit volume, $H^{\prime}$ is the effective magnetic field seen by the particle and $\phi$ represents the angle between the magnetic moment and the external applied field. As the measurement temperature increases in the presence of external field the opposite spins will easily orient parallel to the applied magnetic field with the help of the thermal energy. The magnetization can decrease with increasing temperature only if the thermal energy becomes comparable to the additional anisotropy energy. In our system we expect the particles to be strongly coupled, prohibiting superparamagnetic fluctuations. When the samples are cooled in the presence of external magnetic field, the nanosized particles are already blocked along the direction of external field and the FC curve shows small dependence with temperature.

There are several experimental and theoretical reports on the mysterious origin of magnetism in carbon. The contact induced ferromagnetism in graphite [45, 46] (proximity effect) reported for the meteorite samples, is accounted for the presence of magnetite of size below $90 \mathrm{~nm}$ in close proximity with the carbon atoms. The electron transfer from the half-metal magnetite to the semi-metal graphite to equalize the chemical potential induces complete spin-polarization in the surrounding graphite layers. In order to understand whether iron carbide inclusions also spin polarizes the charge carriers similar to magnetite, it is important to know its electronic structure [47]. The overall characteristics of the electronic energy spectrum are similar to those of pure 
iron with the density of states at the fermi level dominated by localized iron d-states and having different densities of spin-up and spin-down electrons. A net transfer of spin across the metal-carbon interface would result, but complete spin polarization (as in the case of magnetite) will not be possible. Even if spin transfer across the interface was the cause for the excess magnetization seen in the $980^{\circ} \mathrm{C}$ compositions, it should surely be seen in the compositions prepared at $900^{\circ} \mathrm{C}$ and containing higher concentration of iron carbide. But what we observe is an antiferromagnetic interaction between the localized carbon spins in most of the C-Fe compositions except for the CFe05900 composition.

The other possible reasoning would be to include the effect of hydrogen atoms. In the case of nanographitic strips, theoretical calculation shows unusual high density of states, which lead to local moments at the strip edges and with suitable stacking, antiferromagnetism [48]. Hydrogen bonding with the dangling bonds gives rise to differences in spin densities and the theoretical calculation predicts the existence of ferromagnetism $[49,50]$. The hydrogen content estimated from the microanalyses, given in Table I, does not show any sharp rise with the preparation temperature. The hydrogen content in the compositions were approximately the same, except for CFe05980 sample $(\sim 1.97 \%)$.

There are several reports on the size dependent enhanced magnetization reported for nanoscale magnetic particles involved ensembles. For the nanoscale particles, enhancement in the magnetization values are seen at temperatures below their blocking temperature. Significant enhancement of magnetic moment per Fe atom in the range 2.11-2.31 $\mu_{B}$ are reported for fcc-structured Fe containing alloys [51]. Theoretical calculation of iron clusters embedded in cobalt [52] and metallic environment [53] showed enhancement in the iron moment as compared to that of a free iron cluster of similar size. Another theoretical study [54] showed that, the cluster size and the local environment effects the magnetic moments of pure Fe clusters. In the case of ferromagnetic particles in contact with the carbon species, spin polarized electrons from the ferromagnetic contacts are injected into the surrounding carbon shells, and the electron spins are coherently transported, i.e, not flipped for long distances [55, 56, 57].

The observed enhanced magnetic moments at room temperature in the lower iron concentration C:Fe compositions are the combined effect of the nanosized particles and the surrounding carbon shell. The hydrogen bonds with the carbon would also favour in stabilizing the spins, leading to excess magnetic moment at room temperature. The Curie-Weiss contribution to the temperature dependence of magnetization is in accordance with the intrinsic property of the carbon form [38, 58, 59]. The intrinsic magnetic moment in carbon materials is attributed to the effect of dangling bonds created by the presence of various defect centers (adatoms, vacancy, zigzag structures).

\section{Summary}

The synthesis of iron carbide nanoparticles embedded in the carbon matrix has been effectively carried out using thermal assisted CVD. The method yielded reasonably well 
dispersed spherical particles. Möossbauer spectroscopy investigation on the prepared compositions showed the effect of particle size and carbon species on the magnetic hyperfine splitting parameters. The enhanced magnetization and hysteresis values are associated with the morphology of the ultrafine magnetic inclusions. The study reflects the fact that preparation condition and the degree of ordering of the carbon matrix surrounding the nanosized ferromagnetic particles influences the ferromagnetic behaviour of the as-prepared C:Fe compositions.

\section{Acknowledgments}

We gratefully acknowledge Central Facility, Department of Physics, Indian Institute of Science for the XRD measurements; Institute Nanoscience Initiative for the TEM measurements; SQUID Magnetometer Faciliy, Division of Chemical Sciences, Indian Institute of Science, for the magnetization measurements. Grateful thanks to Sheshank Shekar and Ved Prakash Arya for their help during the experimental works.

\section{References}

[1] Xiao G and Chien CL 1987 Appl. Phys. Lett. 511280

[2] Liou SH and Chien CL 1988 Appl. Phys. Lett. 52512

[3] Chen C, Hitakami O, Okamoto S and Shimada Y 1999 J. Appl. Phys. 862161

[4] Li XY, Kong LT and Liu BX 2005 Phys. Rev. B 72054118

[5] Xie Yuannan and Blackman JA 2002 Phys. Rev. B 66085410

[6] Fernando GW, Watson RE, Weinert M, Kocharian AN, Ratnaweera A and Tennakone K 2000 Phys. Rev. B $\mathbf{6 1} 375$

[7] McHenry ME, Majetich SA, Artman JO, DeGraef M and Staley SW 1994 Phys. Rev. B 4911358

[8] Scott JHJ and Majetich SA 1995 Phys. Rev. B $\mathbf{5 2} 12564$

[9] Tartaj P, Morales MP, Veintemillas-Verdaguer S, Gonzalez- Carreno T and Serna CJ 2003 J. Phys. D: Appl. Phys. 36 R182

[10] Zhao XQ, Liang Y, Hu ZQ and Liu BX 1996 J. Appl. Phys. 805857

[11] Masuda M, Maeda K, Kobayashi T, Shiomi S, Fujiwara Y and Saito Y 2000 J. Appl. Phys. 39 L733

[12] Scott JHJ and Majetich SA 1995 Phys. Rev. B 5212564

[13] Zhao XQ, Liang Y, Hu ZQ and Liu BX 1996 J. Appl. Phys. 80, 5857

[14] Grimes CA, Horn JL, Bush GG, Allen JL and Eklund PC 1997 IEEE Trans. Magn. 333736

[15] Grimes CA, Qian D, Dickey EC, Allen JL and Eklund PC 2000 J Appl. Phys. 875642

[16] Denes FS, Manolache S, Ma YC, Shamamian V, Ravel B and Prokes S 2003 J. Appl. Phys. 94 3498

[17] Hayashi K, Ohsugi M, Kamigaki M, Xia B, and Okuyamab K 2002 Electrochem. Solid-State Lett. 5 J9-J12

[18] Goodwin TJ, Yoo SH, Matteazzi P and Groza JR 1997 Nanostruct. Mater. 8559

[19] Yumoto H, Nagamine Y, Nagahama J and Shimotomai M 2002 Vacuum 65527

[20] Wilson JL, Poddar P, Frey NA, Srihanth H, Mohomed K, Harmon JP, Kotha S and Wachsmuth J 2004 J. Appl. Phys. 951439

[21] Kim HM, Kim K, Lee CY, Joo J, Cho SJ, Yoon HS, Pejakovic DA, Yoo JW and Epstein AJ 2004 Appl. Phys. Lett. 84589

[22] Sajitha EP, Prasad V, Subramanyam SV, Eto S, Takai K and Enoki T 2004 Carbon 422815

[23] Kumaran JTT and Bansal C 1991 Nucl. Instr. And Methods in Phys. Res. B 61357 
[24] Lümmen N and Kraska T 2004 Nanotechnology 15525

[25] Bronikowski MJ, Willis PA, Colbert DT, Smith KA and Smalley RE 2001 J. Vac. Sci. Technol. A 19(4) 1800

[26] Ermakova MA, Ermakov DY, Chuvilin AL and Kuvshinor GS 2001 J. Catal. 201183

[27] Kuznetsov VL, Usoltseva AN, Chuvilin AL, Obraztsova ED and Bonard JM 2001 Phys. Rev. B 64235401

[28] Rancourt DG and Ping JY 1991 Nucl. Instr. and Meth. Phys. Res. B 5885

[29] Ron M and Mathalone Z 1971 Phys. Rev. B 4774

[30] Hayashi M, Tamura I, Fukano Y, Kanemaki S and Fujio Y 1980 J. Phys. C: solid St. Phys. 13681

[31] Hofer JE and Cohen Em 1959 J. Amer. Chem. Soc 811576

[32] Esquinazi P and Höhne R 2005 J. Magn. Magn. Mater 290-291 20

[33] Stoner EC and Wohlfarth EP 1948 Philos. Trans. R. Soc. London, Ser. A. 240599

[34] Kittel C 1976 Introduction to Solid State Physics 5th ed. (Wiley, New York)

[35] Holmes M, O'Grady K and Popplewell J 1990 J. Magn. Magn. Mater. 8547

[36] Söffge F and Schmidbauer E 1981 J. Magn. Magn. Mater. 2454

[37] Dubovik VM, Martsenyuk MA and Martsenyuk NM 1995 J. Magn. Magn. Mater. 150105

[38] Mattis DC 2005 Phys. Rev. B 71144424

[39] Crespo P, Gonzalez JM, Hernando A and Yndurain FJ 2004 Phys. Rev. B 69012403

[40] Argyle BE, Charap SH and Pugh EW, Phys. Rev 1322051

[41] Zheng M, Menon L, Zeng H, Liu Y, Bandyopadhyay S, Kirby RD and Sellmyer DJ 2000 Phys. Rev. B 6212282

[42] Zhang D, Klabunde KJ and Sorensen CM 1998 Phys. Rev. B 5814167

[43] Torre ED, Bennett LH and Watson RE 2005 Phys. Rev. Lett. 94147210

[44] Shenoy VB 2005 Phys. Rev. B 71125431

[45] Coey J, Venkatesan M, Fitzgerald C, Douvalis A and I. Sanders I 2002 Nature 420156

[46] Höhne R, Ziese M and EsquinaziP 2004 Carbon 423109

[47] Häglund J, Grimvall G and Jarlborg T 1991 Phys. Rev. B 442914

[48] Harigaya K 2001 J. Phys.: Condens. Matter 131295

[49] Kusakabe K and Maruyama M 2003 Phys. Rev. B 67092406

[50] Ruffieux P, Gröning O, Schwaller P, Schlapbach L and Gröning P 2000 Phys. Rev. Lett. 844910

[51] Li XY, Kong LT and Liu BX 2005 Phys. Rev. B 72054118

[52] Xie Y and Blackman JA 2002 Phys. Rev. B 66085410

[53] Fernando GW, Watson RE, Weinert M, Kocharian AN, Ratnaweera A and Tennakone K 2000 Phys. Rev. B 61375

[54] Press MR, Liu F, Khanna SN and Jena P 1989 Phys. Rev. B 40399

[55] Tsukagoshi K, Alphenaar BW and Ago H 1999 Nature 401572

[56] Kang YJ, Choi J, Moon CY and Chang KJ 2005 Phys. Rev. B 71115441

[57] Fujima N and Oda T 2005 Phys. Rev. B $\mathbf{7 1} 115412$

[58] Rode AV, Gamaly EG, Christy AG, Fitz Gerald JG, Hyde ST, Elliman RG et. al. 2004 Phys. Rev. $B \mathbf{7 0} 054407$

[59] Shibayama Y, Sato H, Enoki T and Endo M 2000 Phys. Rev. Lett. 841744 
Table 1. Effect of precursor concentration and deposition temperature on the structural properties of the C:Fe compositions.

\begin{tabular}{cccccc}
\hline Compositions & $\begin{array}{c}\mathrm{Fe}^{a} \\
(\%)\end{array}$ & $\begin{array}{c}\text { Hydrogen } \\
(\%)\end{array}$ & $\begin{array}{c}d \\
(\AA)\end{array}$ & $\begin{array}{c}\mathrm{g} \\
(\%)\end{array}$ & $\begin{array}{c}L_{C} \\
(\mathrm{~nm})\end{array}$ \\
\hline $\mathrm{CFe} 05900$ & 8.9 & 0.94 & 3.418 & 25.8 & 5.87 \\
$\mathrm{CFe} 10900$ & 9.3 & 0.93 & 3.371 & 80.5 & 18.99 \\
$\mathrm{CFe} 05980$ & 4.6 & 1.97 & 3.424 & 18.6 & 5.29 \\
$\mathrm{CFe} 10980$ & 8.6 & 0.95 & 3.419 & 24.4 & 5.11 \\
\hline
\end{tabular}

a. Percentage error is between $1.2 \%$ and $2 \%$.

Table 2. Mössbauer parameters for the C:Fe compositions. $H_{h f}(\mathrm{~T})$ is the magnetic hyperfine field, $\delta(\mathrm{mm} / \mathrm{s})$ is the isomer shift, and $\Delta(\mathrm{mm} / \mathrm{s})$ is the electric quadrupole shifts.

\begin{tabular}{ccccc}
\hline sample & $H_{h f}(\mathrm{~T})$ & $\delta(\mathrm{mm} / \mathrm{s})$ & $\Delta(\mathrm{mm} / \mathrm{s})$ & Area $(\%)$ \\
\hline $\mathrm{CFe} 05900$ & $20.33(0)$ & $0.18(3)$ & $0.00(6)$ & $94 \pm 1$ \\
& & $0.075(6)$ & $0.00(0)$ & $6 \pm 1$ \\
$\mathrm{CFe} 10900$ & $20.40(4)$ & $0.19(0)$ & $0.00(0)$ & $87 \pm 1$ \\
& & $0.76(2)$ & $1.09(1)$ & $13 \pm 1$ \\
$\mathrm{CFe} 05980$ & $20.23(9)$ & $0.21(9)$ & $0.00(6)$ & 100 \\
$\mathrm{CFe} 10980$ & $20.20(0)$ & $0.21(2)$ & $0.00(4)$ & 100 \\
\hline
\end{tabular}

Table 3. Effect of precursor concentration and deposition temperature on the magnetic properties of the C:Fe compositions.

\begin{tabular}{lllllll}
\hline \multirow{2}{*}{ sample } & \multicolumn{2}{c}{$M_{S}$} & $(\mathrm{emu} / \mathrm{g})^{\mathrm{a}}$ & & \multicolumn{2}{c}{$S=\frac{M_{R}}{M_{S}}$} \\
\cline { 2 - 3 } & $300 \mathrm{~K}$ & $10 \mathrm{~K}$ & & $\begin{array}{l}M_{S-c a l} \\
(\mathrm{emu} / \mathrm{g})\end{array}$ & $(300 \mathrm{~K})$ & $(10 \mathrm{~K})$ \\
\hline $\mathrm{CFe} 05900$ & 9.21 & 11.58 & 15.04 & 0.25 & 0.43 \\
$\mathrm{CFe} 10900$ & 8.9 & 10.58 & & 15.72 & 0.16 & 0.39 \\
$\mathrm{CFe} 05980$ & 8.3 & 10.37 & & 7.27 & 0.32 & 0.49 \\
$\mathrm{CFe} 10980$ & 12.02 & 14.97 & & 14.53 & 0.28 & 0.50 \\
\hline
\end{tabular}

a Magnetization values at $1.5 \mathrm{~T}$.

Table 4. The fitting parameters of the C:Fe compositions obtained using Eq. 4. ( $N_{l o c}$, is calculated using Eq. 4)

\begin{tabular}{cccccc}
\hline sample & $\begin{array}{c}M_{0} \\
\mathrm{emu} / \mathrm{g}\end{array}$ & $\begin{array}{c}B \\
\mathrm{~K}^{2 / 3} \times 10^{-5}\end{array}$ & $\begin{array}{c}C \\
\mathrm{emu} \mathrm{K} / \mathrm{g}\end{array}$ & $\begin{array}{c}\theta \\
\mathrm{K}\end{array}$ & $\begin{array}{c}N_{\text {loc }} \\
\times 10^{24}\end{array}$ \\
\hline $\mathrm{CFe} 05900$ & 10.5 & 3.8 & 5.2 & 0.37 & 8.35 \\
$\mathrm{CFe} 10900$ & 10.5 & 3.6 & 6.25 & -1.68 & 10.0 \\
$\mathrm{CFe} 05980$ & 9.76 & 4.1 & 10.5 & -3.53 & 16.9 \\
$\mathrm{CFe} 10980$ & 14.96 & 3.8 & 6.75 & -0.89 & 10.1 \\
\hline
\end{tabular}




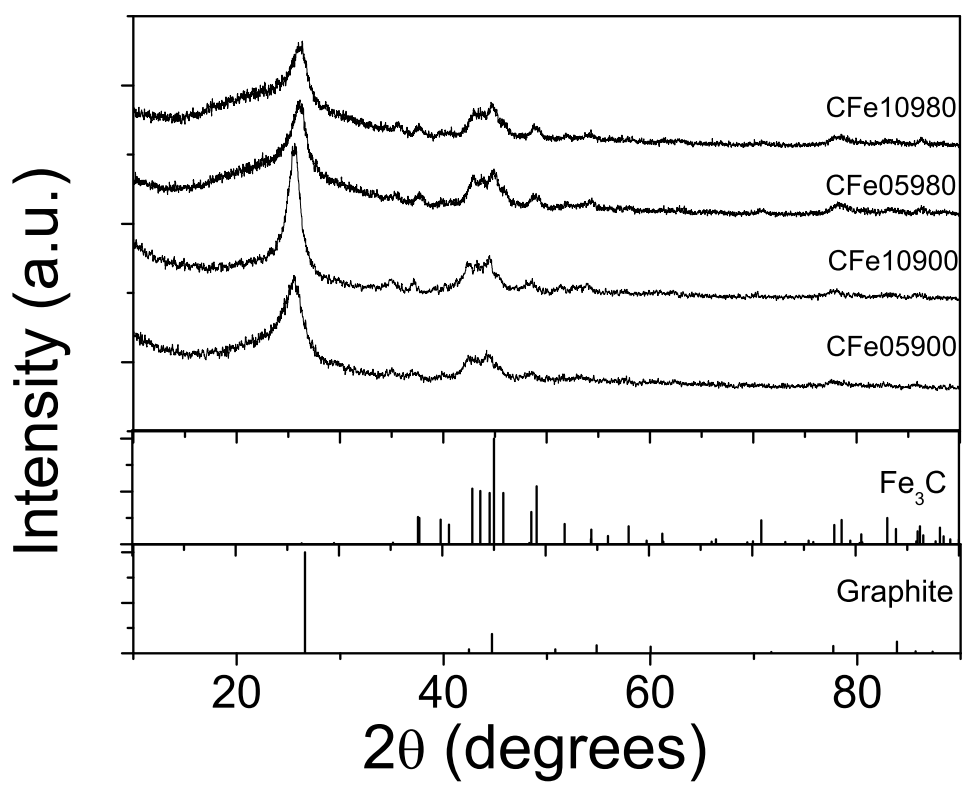

Figure 1. X-ray diffraction pattern for the as-prepared C:Fe compositions, as a functions of preparation temperature and ferrocene content. Standard XRD peaks for iron carbide and graphite (from JCPDS card) are also plotted for reference. 

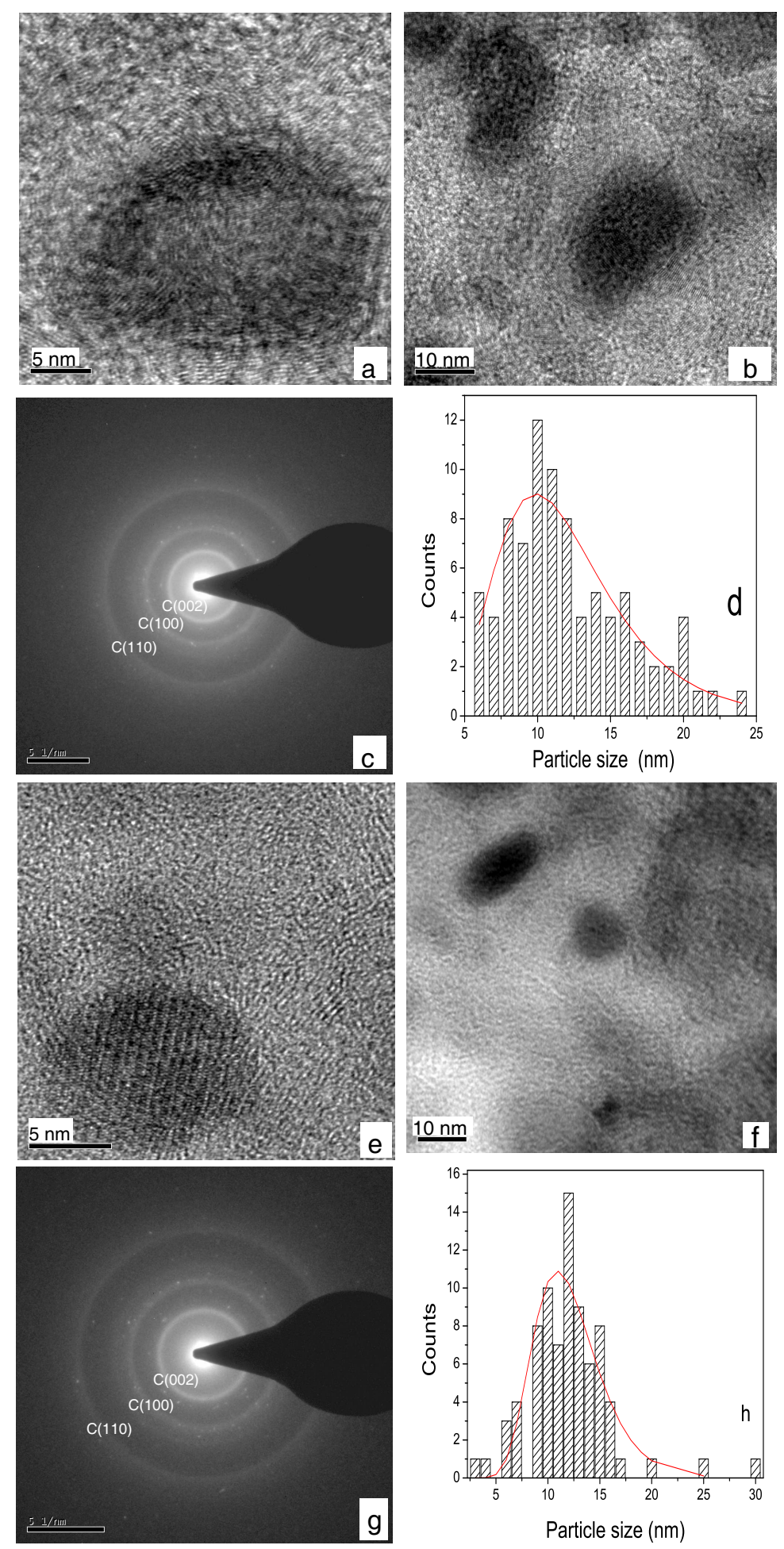

Figure 2. (a), (b) TEM micrographs of as-prepared CFe05980 composition; (c) the corresponding SAED pattern and (d) the particle size histogram adjusted by a log-normal curve (solid line). (e), (f) TEM micrographs of as-prepared CFe10980 composition; (g) the corresponding SAED pattern and (h) the particle size histogram adjusted by a log-normal curve (solid line). 


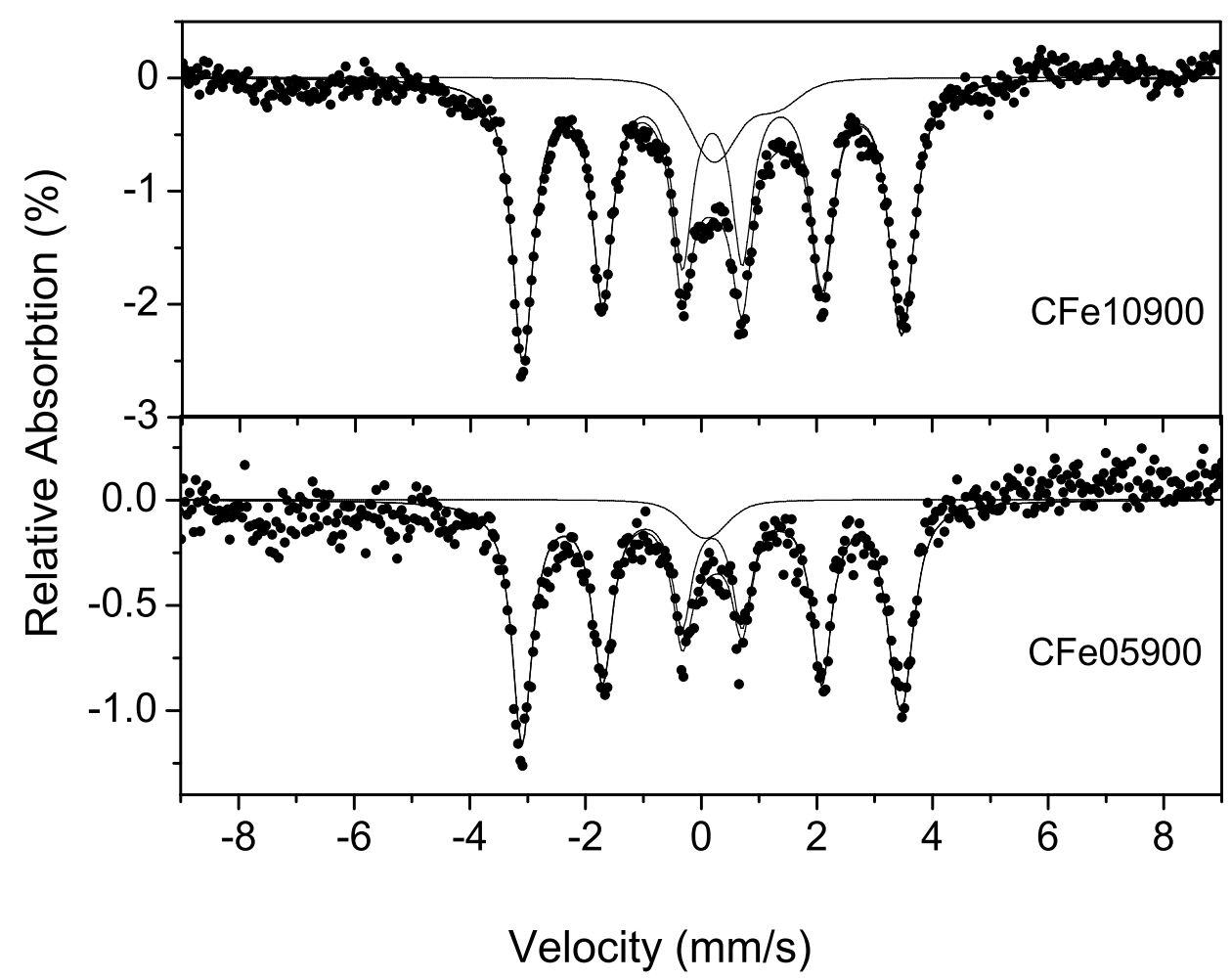

Figure 3. Room-temperature Mössbauer spectra of the C:Fe compositions prepared at $900^{\circ} \mathrm{C}$. The line is the least-square fit to the data (dots)
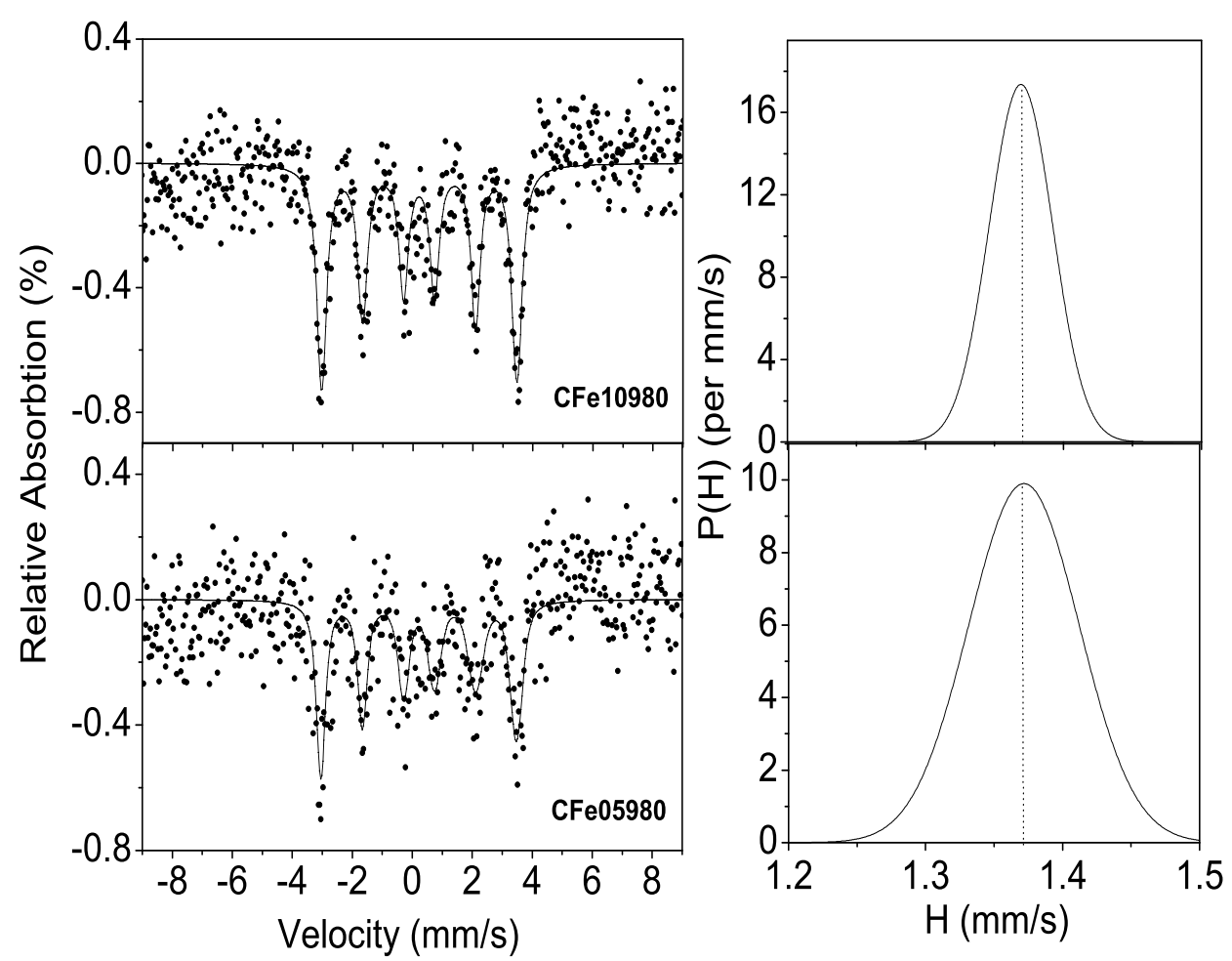

Figure 4. Room-temperature Mössbauer spectra of the C:Fe compositions prepared at $980^{\circ} \mathrm{C}$. The line is the least-square fit to the data (dots) 


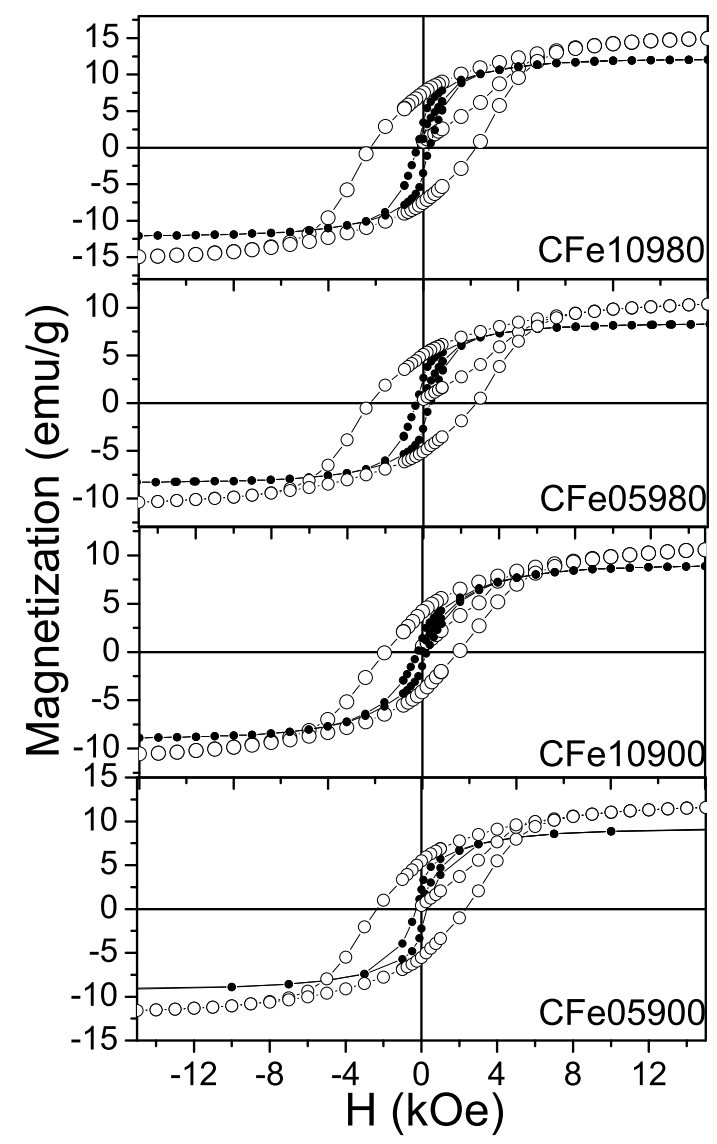

Figure 5. Hysteresis loops at $300 \mathrm{~K}(\bullet)$ and $10 \mathrm{~K}(\circ)$ for the as-prepared C:Fe compositions.

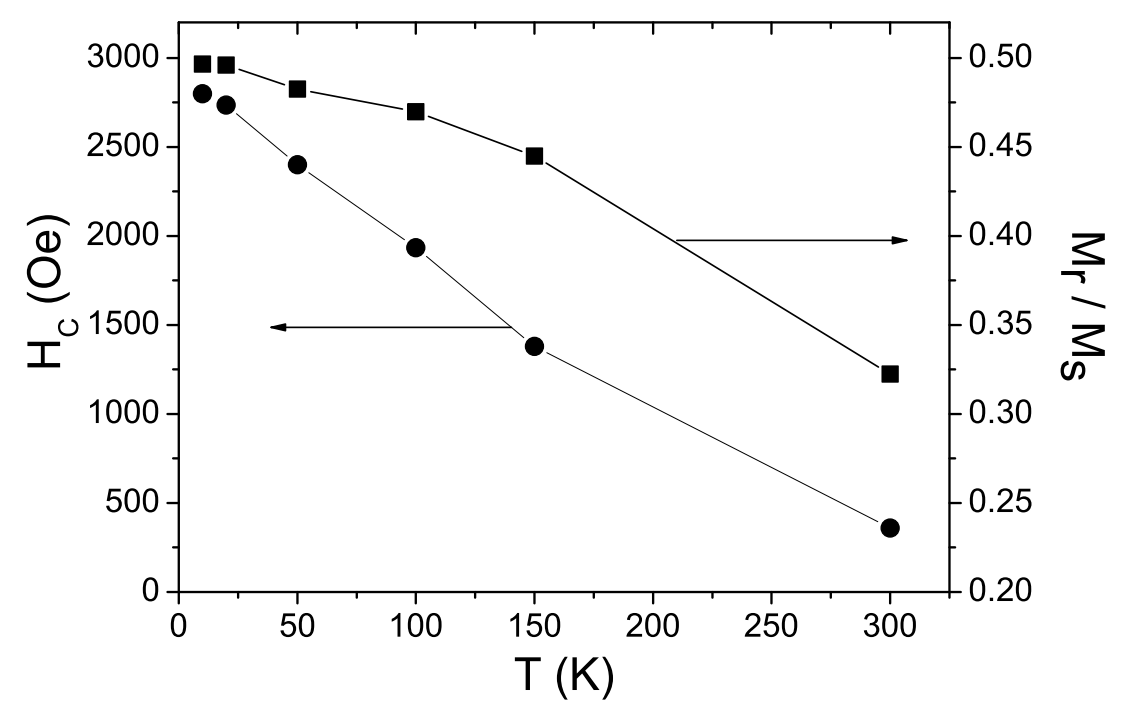

Figure 6. Coercivity and remanence ratio as a function of temperature for $\mathrm{CFe} 05980$. 


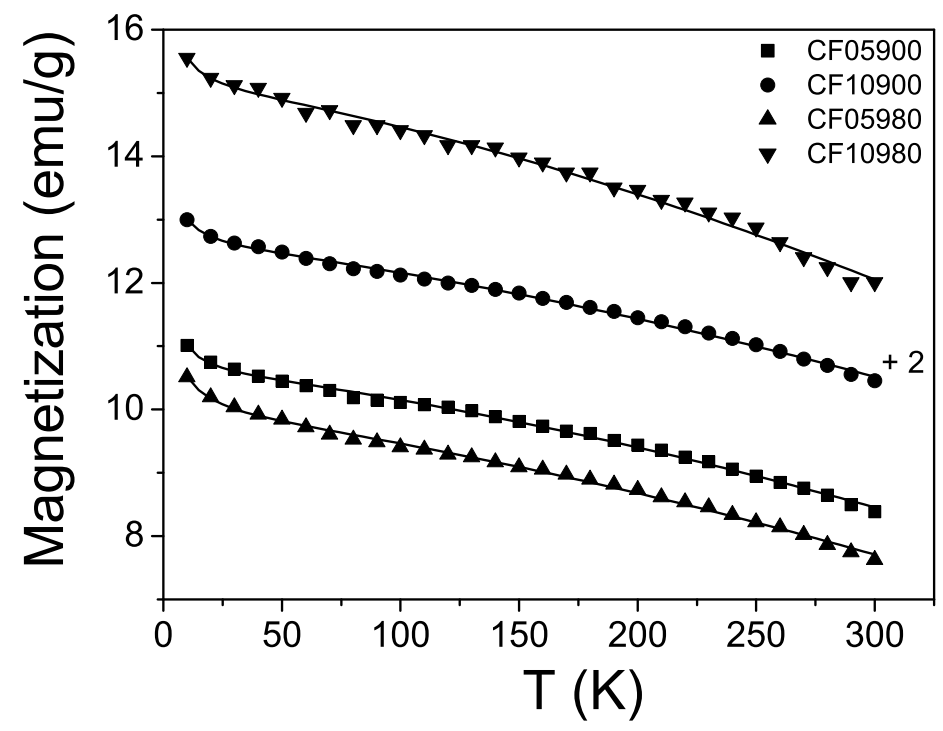

Figure 7. Magnetization versus temperature plot for the C:Fe compositions. The discrete points are the data sets and the continuous line is the fit to the Eq. 4.

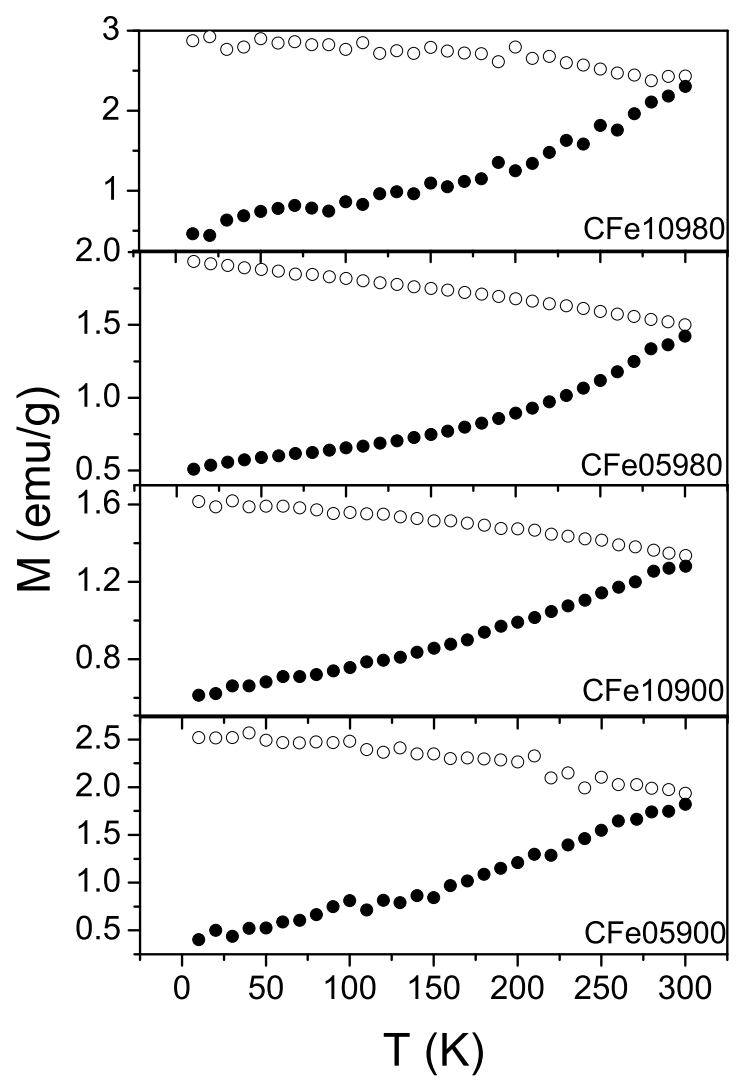

Figure 8. The zero-field cooling $(\bullet)$ and field cooling (o) curve for the $\mathrm{C}: \mathrm{Fe}$ compositions. 